\title{
DESALUMINAÇÃO DO ZEÓLITO OFRETITA POR EFEITO DA RADIAÇÃO $\gamma$
}

\section{A. P. Carvalho e M. Brotas de Carvalho}

Faculdade de Ciências - Universidade de Lisboa - Departamento de Química e Bioquímica - Rua Ernesto de Vasconcelos - Bloco C1 - 1700 Lisboa - Portugal

Recebido em 23/7/97; aceito em 7/4/98

\begin{abstract}
DEALUMINATION OF OFFRETITE THROUGH $\gamma$-IRRADIATION. Protonic offretite samples were modified by $\gamma$-irradiation under different conditions of humidity. The results of infrared spectroscopy and $X$-ray diffraction reveal that the presence of water is essential to promote dealumination, as the samples irradiated in vacuum only show crystallinity loss. The degree of dealumination is, mainly, controlled by the $\gamma$-irradiation dose while the textural modifications depend also on the percentage of water.
\end{abstract}

Keywords: dealumination; offretite; $\gamma$-irradiation.

\section{INTRODUÇÃO}

As propriedades dos zeólitos como catalisadores, adsorventes e permutadores iônicos, estão diretamente relacionadas com o teor em alumínio da rede cristalina, constatando-se, por exemplo, que o aumento da razão $\mathrm{Si} / \mathrm{Al}$ da rede zeolítica se traduz numa maior estabilidade térmica e em alterações significativas da acidez destes materiais.

Na medida em que a maioria das estruturas zeolíticas só pode ser sintetizada num intervalo muito estreito de razões $\mathrm{Si} / \mathrm{Al}$, revestem-se de grande importância os tratamentos de desaluminação que, como o nome indica, permitem remover uma fração dos átomos de alumínio da estrutura zeolítica, sem que tal afete de forma muito acentuada a rede cristalina das amostras.

A primeira referência a este tipo de tratamentos pós-síntese é um estudo realizado nos anos 60 por Barrer e Makki ${ }^{1}$ sobre a modificação do zeólito clinoptilolita por tratamentos ácidos. Desde este trabalho pioneiro encontram-se propostos na literatura diversos outros processos de desaluminação de estruturas zeolíticas, sendo os tratamentos hidrotérmicos (calcinações a temperaturas superiores a $773 \mathrm{~K}$, em regime de steaming ou self-steaming, isto é, introduzindo ou não um fluxo contínuo de vapor de água no sistema reaccional) $)^{2,3}$ e os tratamentos com $\mathrm{SiCl}_{4}{ }^{4}$ os mais vulgarmente usados. Dos restantes salientam-se reações com EDTA ${ }^{5}$ ou outros agentes quelantes ${ }^{6}$ e tratamentos com soluções de $\left(\mathrm{NH}_{4}\right)_{2} \mathrm{SiF}_{6}{ }^{7}$, cuja aplicação é relativamente frequente, e reações com $\mathrm{F}_{2}{ }^{8}$, com compostos fluorados ${ }^{9,10}$ e compostos clorados ${ }^{11,12}$, que são empregues de modo esporádico.

As estruturas zeolíticas podem ainda ser modificadas por efeito da radiação $\gamma$, encontrando-se, de fato, na literatura, alguns trabalhos em que por este processo se promovem alterações das propriedades ácidas das estruturas irradiadas ${ }^{13-17}$. Revendo estes estudos conclui-se que, por um lado, nenhum deles teve em vista a desaluminação das estruturas e, por outro, constata-se que em nenhum dos casos as estruturas foram irradiadas em atmosfera de umidade.

Considerando que a presença de água é determinante para promover a desaluminação das estruturas zeolíticas por tratamentos térmicos, o trabalho que agora se apresenta teve por objetivo realizar um estudo preliminar sobre o efeito da radiação $\gamma$, nomeadamente do calor gerado por esta radiação, sobre estruturas zeolíticas, em diferentes condições de umidade. A estrutura selecionada para este estudo foi a do zeólito ofretita, dado que este trabalho se enquadra num estudo mais vasto sobre a desaluminação desta estrutura zeolítica ${ }^{18-20}$, dispondose assim de um conjunto de resultados que permite confrontar as características das amostras irradiadas com as apresentadas pelas estruturas desaluminadas por processos mais usuais, nomeadamente, tratamentos hidrotérmicos.

O zeólito ofretita apresenta uma rede cristalina de simetria hexagonal, sendo o sistema poroso constituído por dois tipos de canais: grandes canais retilíneos (aberturas de 12 átomos de oxigênio) interligados por cavidades gmelinita (aberturas elípticas de 8 átomos de oxigênio), cuja sucessão origina canais sinuosos e perpendiculares aos anteriores ${ }^{21-23}$. Estas características estruturais conferem à ofretita a propriedade de seletividade de forma: enquanto que moléculas com diâmetros críticos relativamente pequenos, como os hidrocarbonetos lineares, tem acesso aos dois tipos de canais, moléculas mais volumosas, como por exemplo os xilenos, difundem-se apenas nos grandes canais.

\section{PARTE EXPERIMENTAL}

\section{Preparação das amostras}

A amostra de ofretita protônica, HOFF, que serviu de base a este estudo foi obtida da correspondente forma K,TMA,OFF (TMA - íon tetrametilamônio) seguindo a protocolo descrito na ref. 18 e apresenta a seguinte composição da célula unitária, estimada a partir dos resultados de análise elementar:

$$
\mathrm{K}_{0.50} \mathrm{H}_{2.40} \mathrm{Al}_{2.9} \mathrm{Si}_{15.1} \mathrm{O}_{36}
$$

Para proceder à irradiação introduziram-se amostras de cerca de $200 \mathrm{mg}$ em ampolas de pyrex que foram seladas nas seguintes condições:

A - sob vácuo, após desgaseificação a $673 \mathrm{~K}$ durante 3 horas com vácuo inferior a $10^{-5}$ torr.

B - numa atmosfera de vapor de água, correspondente à tensão de vapor de 23 torr.

$\mathrm{C}$ - na presença de água, numa proporção de volume de água/ massa de zeólito de $0.5 \mathrm{~cm}^{3} \mathrm{~g}^{-1}$.

Nestas condições as amostras, inicialmente à temperatura ambiente, foram expostas a doses de radiação $\gamma$ entre 300 e $2100 \mathrm{kGy}$, com uma taxa de dose de $40 \mathrm{kGy} \mathrm{h}^{-1}$, à temperatura ambiente, usando uma fonte de cobalto-60. Após irradiação, e seguindo os procedimentos adotados por outros autores ${ }^{13,14}$, as amostras permaneceram seladas durante 3 semanas antes de se proceder à sua caracterização.

Na continuação as amostras irradiadas são designadas pelas letras $\mathrm{A}, \mathrm{B}$ ou $\mathrm{C}$, indicativas das condições de umidade em que decorreu a irradiação, seguidas da dose de radiação a que foram expostas. 


\section{Técnicas de caracterização}

Os difratogramas de raios $X$, para valores de $2 \theta$ entre 5 e $40^{\circ}$, foram obtidos à temperatura ambiente num difractômetro Philips 1820 , usando a radiação $\mathrm{K} \alpha$ do Cobre isolada da $\mathrm{K} \beta$ por intermédio de um filtro de níquel. Antes de se proceder a esta análise as amostras foram colocadas num hidratador $35 \%$ de umidade relativa) durante, pelo menos, 16 horas. Com base nestes resultados estimou-se a percentagem de cristalinidade das amostras, $C_{R X}$, pela razão entre as somas das alturas dos picos correspondentes aos índices de Miller (210), (211), (102) e (212) da amostra em estudo e da HOFF, estrutura que se tomou como padrão e que, portanto, se considerou $100 \%$ cristalina. O parâmetro $a$ da célula unitária foi determinado a partir de todas as reflexões no intervalo de $2 \theta$ entre 5 e $40^{\circ}$ pelo método dos mínimos quadrados ${ }^{24}$.

Os espectros de infravermelho na região entre 1300 e $300 \mathrm{~cm}^{-1}$ foram realizados à temperatura ambiente num espectrofotômetro HITACHI 250-50, com uma resolução de $2 \mathrm{~cm}^{-1}$. As amostras foram analisadas sob a forma de pastilhas, preparadas a partir de misturas onde a amostra de zeólito foi diluída em $\mathrm{KBr}$ na proporção de $1 / 300$.

As isotermas de adsorção de nitrogênio a $77 \mathrm{~K}$ foram determinadas numa instalação volumétrica convencional equipada com um sensor de pressão MKS-Baratron (0-1000 torr). Antes de se efetuarem estes ensaios os sólidos foram submetidos a um tratamento de desgaseificação a $673 \mathrm{~K}$ durante 3 horas, sob vácuo dinâmico melhor que $10^{-5}$ torr. Os resultados de adsorção são expressos em volume de nitrogênio, considerado líquido $\left(\rho_{\mathrm{N} 2}=0.808 \mathrm{~g} \mathrm{~cm}^{-3}\right)$, por massa de adsorvente desgaseificado.

\section{RESULTADOS E DISCUSSÃO}

A caracterização das amostras por espectroscopia de infravermelho foi efetuada apenas na designada região T-O-T, 1300$300 \mathrm{~cm}^{-1}$, isto é, na região onde as bandas de absorção refletem as vibrações das unidades primárias de construção das estruturas zeolíticas, os tetraedros $\mathrm{TO}_{4}(\mathrm{~T}=\mathrm{Si}$ ou $\mathrm{Al})$. Assim, na Tabela 1 apresentam-se os valores dos números de onda das bandas de estiramento assimétrico, $v_{1}$, e simétrico, $v_{2}$, das amostras HOFF e estruturas irradiadas. Os valores de $v_{2}$ correspondem a máximos de bandas muito bem definidas e estreitas pelo que se considera que nas condições em que foram obtidos os espectros a precisão destes valores é igual ou inferior a $1 \mathrm{~cm}^{-1}$. A evolução dos valores que caracterizam as amostras irradiadas relativamente aos da amostra inicial comprova a desaluminação das estruturas, pois o decréscimo progressivo do número de alumínios da rede zeolítica, $\mathrm{N}_{\mathrm{Al}}$, reflete-se no deslocamento de ambas as bandas para números de onda mais elevados ${ }^{18,25}$. Este conjunto de resultados mostra também que só foi possível promover a desaluminação parcial da estrutura da ofretita quando a irradiação se processou numa atmosfera úmida. Com efeito, apenas no caso das amostras do tipo $\mathrm{B}$ e $\mathrm{C}$ os valores de $v_{1}$ e $v_{2}$ são significativamente mais elevados do que os apresentados pela amostra HOFF.

Considerando que a desaluminação progressiva das estruturas zeolíticas está associada igualmente à contração dos parâmetros da célula unitária ${ }^{18,25}$, verifica-se que a evolução do parâmetro $a$ das amostras irradiadas é consistente com os dados de infravermelho.

Com base nos valores de $v_{1}$ e $v_{2}$ e nas relações $\mathrm{N}_{\mathrm{Al}}=f\left(v_{1}\right.$ ou $\mathrm{v}_{2}$ ), que foram propostas para este tipo de estrutura zeolítica ${ }^{18}$, é possível analisar quantitativamente os efeitos dos tratamentos efetuados na composição da célula unitária. Assim, na tabela 1 apresentam-se os valores de $\mathrm{N}_{\mathrm{Al}(\mathrm{v} 2)}$ que, como a sua designação indica, foram estimados a partir de valor de $v_{2}$ que caracteriza cada uma das amostras. Não se recorreu à relação $\mathrm{N}_{\mathrm{Al}}=f\left(\mathrm{v}_{1}\right)$ por esta não se encontrar bem definida no intervalo entre 1050 e $1075 \mathrm{~cm}^{-1}$, onde se situam os valores que caracterizam a maioria das amostras irradiadas.
Tabela 1. Números de onda das bandas de estiramento assimétrico, $v_{1}$, e simétrico, $v_{2}$, estimativa do número de alumínios por célula unitária, $\mathrm{N}_{\mathrm{Al}(\mathrm{v} 2)}$, taxa de desaluminação, \%D e parâmetros da célula unitária $a$ e $c$ da HOFF e amostras modificadas por efeito da radiação $\gamma$.

\begin{tabular}{lccccc}
\hline Amostra & $\begin{array}{c}\mathrm{v}_{1} \\
\left(\mathrm{~cm}^{-1}\right)\end{array}$ & $\begin{array}{c}\mathrm{v}_{2} \\
\left(\mathrm{~cm}^{-1}\right)\end{array}$ & $\mathrm{N}_{\mathrm{Al}(\mathrm{v} 2)} *$ & $\% \mathrm{D}^{* *}$ & $\begin{array}{c}a \\
(\AA)\end{array}$ \\
\hline HOFF & 1069 & $792.0 \pm 0.5$ & 2.96 & - & 13.237 \\
A-300 & 1070 & $793.0 \pm 0.5$ & 2.87 & 3.0 & 13.200 \\
A-900 & 1070 & $793.0 \pm 0.5$ & 2.87 & 3.0 & 13.203 \\
B-300 & 1072 & $793.0 \pm 0.5$ & 2.87 & 3.0 & 13.204 \\
B-700 & 1073 & $793.5 \pm 0.5$ & 2.83 & 4.4 & 13.195 \\
B-900 & 1075 & $794.5 \pm 0.5$ & 2.74 & 7.4 & 13.189 \\
B-1500 & 1078 & $795.0 \pm 0.5$ & 2.70 & 8.8 & 13.165 \\
B-2100 & 1080 & $796.5 \pm 0.5$ & 2.57 & 13.2 & 13.137 \\
C-300 & 1072 & $793.0 \pm 0.5$ & 2.87 & 3.0 & 13.192 \\
C-700 & 1073 & $793.5 \pm 0.5$ & 2.83 & 4.4 & 13.190 \\
C-900 & 1075 & $794.0 \pm 0.5$ & 2.79 & 5.7 & 13.186 \\
\hline
\end{tabular}

*estimativa efetuada com base na relação $\mathrm{N}_{\mathrm{Al}}=f\left(\mathrm{v}_{2}\right)^{18} \mathrm{e}$ considerando os valores médios de $v_{2}$.

$$
* * \% \mathrm{D}=\frac{\mathrm{N}_{\mathrm{Al}(\mathrm{v} 2)-\mathrm{HOFF}}-\mathrm{N}_{\mathrm{Al}(\mathrm{v} 2)-\text { Amostra }}}{\mathrm{N}_{\mathrm{Al}(\mathrm{v} 2)-\mathrm{HOFF}}} \times 100
$$

Considerando então os valores de $\mathrm{N}_{\mathrm{Al}(v 2)}$ como representativos do teor em alumínio da célula unitária estimou-se a percentagem de desaluminação das amostras, $\% \mathrm{D}$, estando os resultados expressos na última coluna da tabela 1 . Os valores obtidos mostram que, no limite das condições ensaiadas, se obteve uma estrutura (B-2100) onde foram removidos cerca de $13 \%$ dos átomos de $\mathrm{Al}$ inicialmente presentes na célula unitária. Esta taxa de desaluminação não sendo muito elevada é, no entanto, comparável a valores obtidos para amostras de ofretita desaluminadas por tratamentos de self-steaming e steaming, com percentagem de cristalinidade similares ${ }^{18,26}$. Por outro lado, a confrontação dos resultados apresentados pelas amostras do tipo B e C indica que o número de átomos de Al que é possível remover da rede cristalina da ofretita irradiando a estrutura em presença de umidade parece depender, fundamentalmente, da dose de radiação a que as amostras foram expostas.

A evolução da percentagem de cristalinidade, $C_{R X}$, em função da dose de radiação $\gamma$, que se reproduz na figura 1 , mostra que o aumento da dose de radiação a que a estrutura é exposta acarreta, em qualquer caso, uma maior destruição da estrutura. Observa-se igualmente que para a mesma dose de radiação a perda de cristalinidade é sempre mais acentuada nas amostras irradiadas em vácuo na ausência de umidade (amostras A). Porém, os dados de raios $\mathrm{X}$ obtidos nas amostras tipo $\mathrm{B}$ e $\mathrm{C}$ parecem indicar que uma maior ou menor percentagem de umidade não se reflete na perda de cristalinidade das estruturas, que tal como a taxa de desaluminação, parece depender, essencialmente, da dose de radiação.

As isotermas de adsorção de nitrogênio a $77 \mathrm{~K}$ que se reproduzem na figura 2 exemplificam o efeito da irradiação nas propriedades texturais das amostras.

A análise da configuração de qualquer uma das curvas apresentadas permite constatar que estamos em presença de isotermas reversíveis e predominantemente do tipo I na classificação $\mathrm{BDDT}^{27}$, o que revela o caráter quase exclusivamente microporoso destes sólidos. Confrontando a parte inicial das curvas $\left(\mathrm{p} / \mathrm{p}_{0}<0.4\right)$ verifica-se que para as amostras irradiadas as quantidades adsorvidas são sempre inferiores ao que se observa com a HOFF, sendo as curvas nesta região praticamente sobreponíveis por um deslocamento vertical, o que sugere que a exposição à radiação $\gamma$ acarreta sobretudo uma perda de volume microporoso, e não uma alteração da distribuição de 


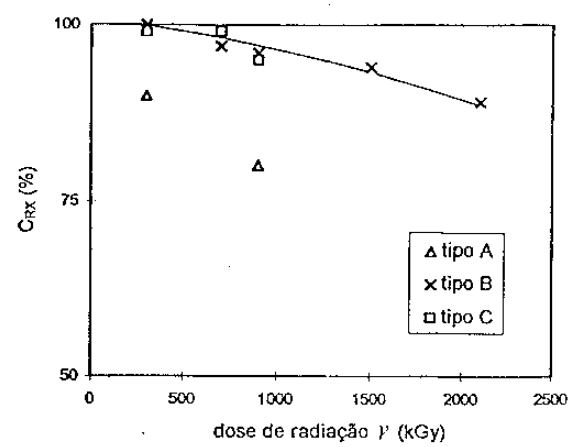

Figura 1. Percentagem de cristalinidade, $C_{R X}$, em função da dose de radiação $\gamma$ a que as amostras dos diversos tipos foram submetidas.

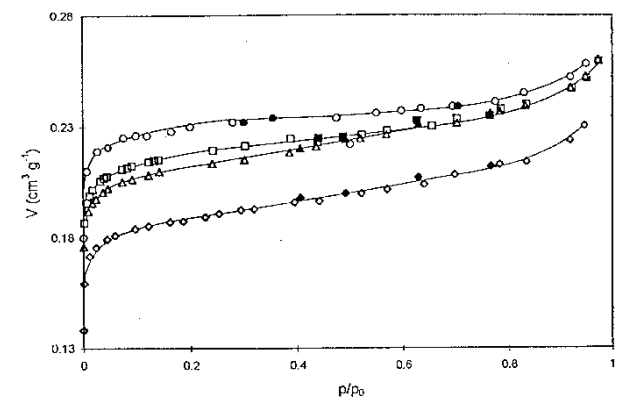

Figura 2. Isotermas de adsorção de nitrogênio a $77 \mathrm{~K} \mathrm{em} \mathrm{:} \mathrm{(O)} \mathrm{HOFF;}$ ( ) A-900; ( ) B-900 e (৩) C-900.

tamanho de microporos. Na região acima de $\mathrm{p} / \mathrm{p}_{0}=0.4$ verifica-se que as isotermas correspondentes às estruturas irradiadas apresentam uma inclinação um pouco mais acentuada que a observada para a curva da amostra inicial, evidenciando a presença de uma percentagem de mesoporosidade ligeiramente mais elevada nas amostras irradiadas.

A micro e mesoporosidade das amostras foi avaliada quantitativamente aplicando, respectivamente, a equação de Dubinin-Raduskevich (DR) ${ }^{28}$ e método $\alpha_{S}{ }^{28}$ e o método de Pierce $^{29}$, estando os resultados expressos na tabela 2 .

Tabela 2. Volumes porosos $\left(\mathrm{cm}^{3} \mathrm{~g}^{-1}\right)$ determinados a partir das isotermas de adsorção de nitrogênio a $77 \mathrm{~K}$ na HOFF e amostras irradiadas.

\begin{tabular}{lccc}
\hline Amostra & $\mathrm{V}_{\mathrm{N} 2(\mathrm{DR})}$ & $\mathrm{V}_{\mathrm{N} 2(\alpha \mathrm{S})}$ & $\mathrm{V}_{\text {MESO }}$ \\
\hline HOFF & 0.22 & 0.22 & 0.02 \\
A-900 & 0.20 & 0.19 & 0.03 \\
B-900 & 0.21 & 0.20 & 0.03 \\
B-1500 & 0.21 & 0.20 & 0.03 \\
B-2100 & 0.21 & 0.20 & 0.03 \\
C-300 & 0.22 & 0.20 & 0.03 \\
C-700 & 0.21 & 0.19 & 0.03 \\
C-900 & 0.19 & 0.18 & 0.03 \\
\hline
\end{tabular}

$\mathrm{V}_{\mathrm{N} 2(\mathrm{DR})}$ - volume microporoso estimado pela aplicação da equação de Dubinin-Raduskevich.

$\mathrm{V}_{\mathrm{N} 2(\alpha \mathrm{S})}$ - volume microporoso estimado pela aplicação do método $\alpha_{S}$ usando-se padrão a isoterma determinada numa sílica não porosa $^{30}$.

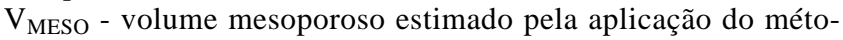
do de Pierce.

A evolução dos volumes microporosos totais estimados pelos dois métodos, $\mathrm{V}_{\mathrm{N} 2(\mathrm{DR})}$ e $\mathrm{V}_{\mathrm{N} 2(\alpha \mathrm{S})}$, são praticamente coincidentes e mostram que a perda de volume microporoso é muito mais acentuada na amostra C-900, concluindo-se que as condições de umidade podem condicionar significativamente as modificações da estrutura, ao contrário do que pareciam indicar tanto os resultados de infravermelho como os de difração de raios $X$, segundo os quais é a dose de radiação e não a percentagem de umidade que determina as características das estruturas modificadas.

Tendo presente que no processo de desaluminação há simultâneamente a formação de espécies extra-rede, cuja mobilidade e localização final dependem da percentagem de umidade, podem então admitir-se situações em que com a mesma rede cristalina seja distinto o acesso ao sistema poroso. Este parece ser o caso exemplificado pelas estruturas B-900 e C900 que resultam praticamente idênticas quando analisadas por infravermelho ou difração de raios $\mathrm{X}$, mas significativamente diferentes em termos de textura microporosa.

\section{CONCLUSÕES}

Os resultados do presente estudo mostram que por efeito da radiação $\gamma$ é possível desaluminar a estrutura da ofretita desde que o processo se efetue numa atmosfera com um certo grau de umidade. Com efeito, nas amostras irradiadas sob vácuo verificou-se apenas uma perda de cristalinidade.

A percentagem de átomos de alumínio removidos da rede cristalina parece depender fundamentalmente da dose da radiação e não das condições de umidade, as quais, no entanto, influenciam a porosidade da estrutura das amostras irradiadas, provavelmente por modificarem a localização final das espécies extra-rede formadas durante o processo.

Com este estudo concluiu-se também que a monitorização do processo de desaluminação pode efetuar-se por espectroscopia de infravermelho ou difração de raios $\mathrm{X}$ pois estas duas técnicas conduzem a resultados concordantes sem, no entanto, evidenciarem diferenças da textura das amostras. Tendo em vista a importância desta propriedade nas amostras desaluminadas tornase pois indispensável recorrer à adsorção de gases, neste caso, nitrogênio a $77 \mathrm{~K}$, para avaliar a eficiência do processo.

Todas estas constatações permitem pois admitir que, desde que se otimizem as condições experimentais, a exposição à radiação $\gamma$ em ambiente de umidade controlada, pode constituir uma alternativa aos diversos métodos de desaluminação de estruturas zeolíticas propostos na literatura.

\section{AGRADECIMENTOS}

Os autores agradecem à Doutora Maria Eduarda Andrade o interesse posto no presente trabalho e a disponibilidade da Unidade Tecnológica de Radioesterilização UTR-GAMA PI de Departamento de Física do Instituto de Tecnologia Nuclear (Sacavém, Portugal) onde as amostras foram irradiadas.

\section{REFERÊNCIAS}

1. Barrer, R. M.; Makki, M. B.; Can. J. Chem. 1964, 42, 1481.

2. Kerr, G. T.; J. Phys. Chem. 1967, 71, 4155.

3. McDaniel, C. V.; Maher, P. K.; In Zeolite Chemistry and Catlysis; J. Rabo Ed., American Chemical Society; Washington, DC, 1976; p. 285.

4. Beyer, H. K.; Belenykaja, I.; In Catalysis by Zeolites; B. Imelik et al., Eds.; Elsevier; Amesterdam,1980; p. 203.

5. Kerr, G. T.; J. Phys. Chem. 1968, 72, 2594.

6. Beaumont, R.; Barthomeuf, D.; J. Catal. 1972, 26, 218.

7. Skeels, G. W.; Breck, D. W.; In Proc. 6th International Zeolite Conference; D. Olson e A. Bisio Eds.; Butherworths; Guilford, 1984; p. 87

8. Lok, B. M.; Izod, T. P. L.; Zeolites 1982, 2, 6.

9. Szostak, R.; American Chemical Society Symp. Series 218; Washington; DC, 1983; p. 41. 
10. Soled, S.; McVicker, G. B.; Ziemiak, J. J.; DeMartin, G.; Yacullo, L.; Strohmaier, K.; Millar, J.; Prep. Am. Chem. Soc., Div. Pet. Chem. 1993, 38, 546.

11. Fejes, P.; Hannus, I.; Kiricsi, I.; Zeolites 1984, 4, 73.

12. Fejes, P.; Kiricsi, I.; Hannus, I.; Schõbel. Gy.; In Zeolites, B. Drzaj et al. Eds.; Elsevier; Amesterdam, 1985; p. 263.

13. El-Nabarawy, Th.; El-Shobaky, G. A.; Dessouki, A. M.; Thermochim. Acta 1987, 111, 249.

14. El-Nabarawy, Th.; El-Shobaky, G. A.; Dessouki, A. M.; Radiat. Phys. Chem. 1989, 33, 23.

15. Li, S. A. H.; Hassan, N. A.; Kira, A. I.; J. Radioanal. Nucl. Chem. 1994, 187, 223.

16. Wichterlová, B.; Nováková, J.; Prásil, Z.; Zeolites 1988 , 8, 117.

17. Sayed, M. B.; Zeolites 1996, 16, 157.

18. Carvalho, A. P.; Brotas de Carvalho, M.; Ramôa Ribeiro, F.; Fernandez, C.; Nagy, J. B.; Derouane, E. G.; Guisnet, M.; Zeolites 1993, 13, 462.

19. Brotas de Carvalho, M.; Carvalho, A. P.; .Ramôa Ribeiro, F.; Florentino, A.; Gnep, N. S.; Guisnet, M.; Zeolites 1994, 14, 217.
20. Carvalho, A. P.; Brotas de Carvalho, M.; Ramôa Ribeiro, F.; Nagy, J. B.; Guisnet, M.; artigo em preparação.

21. Bennett, J. M.; Gard, J. A.; Nature 1965, 214, 1005.

22. Whyte Jr., T. E.; Wu, E. L.; Kerr, G. T.; Venuto, P. B.; J. Catal. 1971, 20, 88.

23. Gard, J. A.; Tait, J. M.; Acta Crystallogr. 1972, $828,825$.

24. Appleman, D. E.; Evans, H. T.; Indexing and LeastSquare Refinement of Powder Diffraction Data, Report No USG-GD-73-003, Washington 1993.

25. Ponthieu, E.; Grange, P.; Joly, J. F.; Raatz, F.; Zeolites 1992, 12, 395.

26. Carvalho, A. P.; Tese de Doutoramento, Universidade de Lisboa, 1996.

27. Brunauer, S.; Deming, L. S.; Deming, W. S.; Teller, E.; J. Amer. Chem. Soc. 1940, 62, 1723.

28. Gregg, S. J.; Sing, K. S. W.; Adsorption Surface Area and Porosity; Academic Press, London 1982.

29. Pierce, C.; J. Phys. Chem. 1953, 57, 149.

30. Payne, D. A.; Sing, K. S. W.; J. Colloid Interface Sci. 1973, 43, 287. 